\title{
CCR7 regulates ANO6 to promote migration of pancreatic ductal adenocarcinoma cells via the ERK signaling pathway
}

\author{
LONG WANG $^{1}$, XIANG-YUN ZHAO ${ }^{1}$, JIN-SHUI ZHU ${ }^{1}$, NI-WEI CHEN ${ }^{1}$, \\ HUI-NING FAN $^{1}$, WEI YANG ${ }^{2}$ and JING-HUI GUO ${ }^{1}$ \\ Departments of ${ }^{1}$ Gastroenterology and ${ }^{2}$ Laboratory, \\ Shanghai Jiao Tong University Affiliated Shanghai Sixth People's Hospital, Shanghai 200233, P.R. China
}

Received March 21, 2017; Accepted November 11, 2017

DOI: $10.3892 / \mathrm{ol} .2018 .8962$

\begin{abstract}
The increase in migratory ability of pancreatic ductal adenocarcinoma cells is a key event in the development of metastasis to the lymph nodes and distant organs. Although the $\mathrm{C}-\mathrm{C}$ motif chemokine receptor 7 (CCR7) and its ligand, $\mathrm{C}-\mathrm{C}$ motif chemokine ligand 21 (CCL21), have been revealed to serve an important role in tumor migration, their precise roles and potential underlying mechanisms remain largely unknown. The present study revealed that overexpression of CCR7 significantly promoted BxPC-3 cell migration, accompanied by the induction of anoctamin 6 (ANO6) expression, indicating that ANO6 is a downstream target of CCR7 signaling. Furthermore, the level of phosphorylated extracellular signal-regulated kinase (ERK) was significantly increased in CCR7-overexpressing BxPC-3 cells, indicating that ERK may be a potential mediator of CCR7-regulated ANO6 expression in BxPC-3 cells. To characterize the receptor-mediated pathway, a specific ERK inhibitor, U0126, was used, which reduced BxPC-3 cell migration and the expression of ANO6. In summary, the results of the present study demonstrate that CCR7 promoted BxPC-3 cell migration by regulating ANO6 expression perhaps via activation of the ERK signaling pathway.
\end{abstract}

\section{Introduction}

Early metastasis to the lymph nodes is a characteristic feature of human pancreatic ductal adenocarcinoma (PDAC). These lymph-node metastases and the disseminated tumor cells in the lymphatic vessels of peri-pancreatic tissues may be the source of local tumor recurrence, which frequently occurs in patients

Correspondence to: Dr Jing-Hui Guo, Department of Gastroenterology, Shanghai Jiao Tong University Affiliated Shanghai Sixth People's Hospital, 600 Yishan Road, Shanghai 200233, P.R. China

E-mail: guohui19820623@126.com

Key words: C-C motif chemokine receptor 7, C-C motif ligand 21, anoctamin 6, extracellular signal-regulated kinase, pancreatic ductal adenocarcinoma following PDAC resection (1). Chemokines, a superfamily of small, secreted peptides characterized by their ability to induce leukocyte migration, together with their receptors, have been revealed to be involved in the migration of cells of the lymphatic system (2). In several types of tumor, chemokine receptors in tumor cells and those secreted by lymphatic endothelial cells (LECs) have been demonstrated to serve a role in the extravasation and homing of circulating tumor cells $(3,4)$.

$\mathrm{C}-\mathrm{C}$ motif chemokine receptor 7 (CCR7), the receptor for the $\mathrm{C}-\mathrm{C}$ motif chemokine ligand 21 (CCL21), is expressed on naive $\mathrm{T}$ cells, memory $\mathrm{T}$ cells, $\mathrm{B}$ cells, mature dendritic cells and certain tumor cells, and is considered to be important in lymphocyte cell trafficking and homing to the lymph nodes (5). In addition, the expression of CCR7 has been reported to promote cancer cell metastasis to lymph nodes in several types of cancer (6-9). Therefore, CCR7 and its ligand may participate in the metastasis of cancer cells of various organ origins. CCR7 was also revealed to be involved in the development and progression of PDAC (10).

Various studies have confirmed the roles of CCL21/CCR7 in tumor development and progression: CCL21/CCR7 is able to upregulate matrix metalloproteinase-9 (MMP-9) in human colon cancer metastasis (11), mediate transforming growth factor $\beta 1$-induced epithelial-mesenchymal transition via crosstalk with nuclear factor- $\kappa \mathrm{B}$ signaling in gastric cancer (12), induce janus kinase 2/signal transducer and activator of transcription 3 phosphorylation in metastatic squamous cell carcinoma of the head and neck (13), upregulate Twist via extracellular signal-regulated kinase (ERK) and phosphoinositide 3-kinase/protein kinase B signaling in PDAC (14) and so on.

The family of anoctamins (ANOs) consists of ten different proteins (TMEM16 genes A-J), ANO1-ANO10. However, there are certain controversies regarding the molecular functions of ANO6 and whether it is a chloride channel, a cation channel or a phospholipid scramblase (15). Notably, the expression of ANO proteins is known to be upregulated in cancer and to be associated with poor patient prognosis (16). Jacobsen et al (17) suggested that ANO6 had an important function in Ehrlich-Lettre ascites (ELA) migration, as part of the migratory 'engine' that determines the speed of cellular migration.

The present study aimed to determine whether ANO6 also contributes to the migration of PDAC cells via the ERK pathway induced by the CCL21/CCR7 axis. 


\section{Materials and methods}

Cell line and reagents. Three human PDAC cell lines, BxPC-3, AsPC-1 and PANC-1, were obtained from American Type Culture Collection (Manassas, VA, USA). Recombinant human CCL21 was obtained from Cyagen Biosciences, Inc. (Santa Clara, CA, USA). The ERK inhibitor U0126 was obtained from Sigma-Aldrich; Merck KGaA (Darmstadt, Germany). The following antibodies were purchased from various sources: Anti-CCR7 (cat no. ab32527; Abcam, Cambridge, UK), anti-ANO6 (cat no. ab156409; Abcam), anti-phosphorylated ERK (pERK) (cat no. KGYT1625; Nanjing KeyGen Biotech Co., Ltd., Nanjing, China) and GAPDH (cat no. ab8245; Abcam). The reverse transcription-quantitative polymerase chain reaction primers for CCR7, ANO6, ERK1/2 and GAPDH were synthesized commercially by Guangzhou RiboBio Co., Ltd. (Guangzhou, China). A cell proliferation kit was obtained from Beijing Dingguo Changsheng Biotechnology Co., Ltd. (Beijing, China). The Migration kit (cat no. \#3422) was obtained from Corning Incorporated (Corning, NY, USA).

Cell culture and transfection. All cells used in the present study were immediately cryopreserved in liquid nitrogen. They were cultured under standard conditions in Dulbecco's Modified Eagle's Medium (Hyclone; GE Healthcare Life Sciences, Logan, UT, USA) supplemented with $10 \%$ fetal bovine serum (FBS; Hyclone; GE Healthcare Life Sciences), $100 \mathrm{U} / \mathrm{ml}$ ampicillin (Hyclone; GE Healthcare Life Sciences) and $100 \mu \mathrm{g} / \mathrm{ml}$ streptomycin (Hyclone; GE Healthcare Life Sciences). The cultures were incubated at $37^{\circ} \mathrm{C}$ in a humidified atmosphere containing $5 \% \mathrm{CO}_{2}$.

Human CCR7 complementary DNA reverse-transcribed from the longest transcript NM_001838 was cloned into the recombined lentiviral vector, recombined by GV358 (pGC-FU-3FLAG-SV40-EGFP-IRES-puromycin; Shanghai GeneChem Co., Ltd., Shanghai, China), pHelper 1.0 (Shanghai GeneChem Co., Ltd.) and pHelper 2.0 (Shanghai GeneChem Co., Ltd.), to create a complete functional overexpression plasmid named LV-CCR7-OE. The empty lentiviral vector was named as LV-GFP. BxPC-3 cells were seeded onto 6-well plates. At $24 \mathrm{~h}$ after seeding, the cells were treated with $5 \mathrm{E}+8$ titration units of lentivirus and harvested at $72 \mathrm{~h}$ for transfection at $37^{\circ} \mathrm{C}$. The transfected BxPC-3 cells were selected if the positive rate of green fluorescent protein (GFP) expression reached $>80 \%$, evaluated by a fluorescence microscope (x200 magnification, Olympus Corporation, Tokyo, Japan). The expression of CCR7 was confirmed using western blotting and reverse transcription-quantitative polymerase chain reaction (RT-qPCR) at $96 \mathrm{~h}$ after transfection in BxPC-3 CCR7-overexpressing cells (BxPC-3-CCR7-GFP cells; OE), blank-vector-transfected negative control (BxPC-3-GFP cells; $\mathrm{NC}$ ) and untransfected control (BxPC-3 cells; CON).

Cell proliferation assay. BxPC-3-CCR7-GFP and BxPC-3-GFP cells were plated at a density of $2 \times 10^{3}$ cells/well in a final assay volume of $100 \mu \mathrm{l}$ per well into 96 -well plates. The cells were incubated for various times $(24,48,72,96$ and $120 \mathrm{~h}$ ) under these conditions. At $4 \mathrm{~h}$ prior to the designed time point, the cells were incubated with MTT. The purple formazan deposits were solubilized in dimethyl sulfoxide.
An automated fluorescence plate reader was used to measure the proliferating cell population at an emission wavelength of $490 \mathrm{~nm}$. BxPC-3-CCR7-GFP and BxPC-3-GFP cells were pre-incubated for $16 \mathrm{~h}$ in the presence or absence of $100 \mathrm{ng} / \mathrm{ml}$ CCL21, which is a chemo-attractant (18), in a humidified, $37^{\circ} \mathrm{C}, 5 \% \mathrm{CO}_{2}$ chamber. To examine the effect of inhibitors, the BxPC-3-CCR7-GFP cells in the absence of CCL21 were pretreated with $10 \mu \mathrm{mol} / 1 \mathrm{U} 0126$ for $2 \mathrm{~h}$.

Cell migration assay. All cell migration assays were performed using a 24-well migration chamber with an 8-mm pore polycarbonate membrane, based on the Boyden chamber principle. Briefly, BxPC-3-CCR7-GFP and BxPC-3-GFP cells were re-suspended in serum-free RPMI 1640 , and $1 \times 10^{5}$ cells were added to the interior of the Transwell inserts in the upper chamber. CCL21 (0 and $100 \mathrm{ng} / \mathrm{ml}$ ) were added to RPMI $1640(500 \mathrm{ml})$ containing $10 \%$ FBS in the lower chamber individually. Following migration for $24 \mathrm{~h}$ at $37^{\circ} \mathrm{C}$, the cells from the top of the membrane were wiped off using cotton swabs whereas the migrated cells (in the bottom chamber) were stained with Giesma for $20 \mathrm{~min}$ at room temperature and washed with distilled water three times. A fluoresence Direct microscopic (Olympus Corporation, Tokyo, Japan) was used at a magnification of $\mathrm{x} 40$ to observe the cells that had migrated to the lower side of the membrane. The absorption value was measured at a wavelength of $570 \mathrm{~nm}$. To examine the effect of inhibitors, the BxPC-3-CCR7-GFP cells in the absence of CCL21 were pretreated with $10 \mu \mathrm{mol} / 1 \mathrm{U} 0126$ for $2 \mathrm{~h}$.

$R T-q P C R$. Total cellular mRNA, purified using TRIzol (Shanghai Pufei Biotechnology Co., Ltd., Shanghai, China), was subsequently reverse transcribed to cDNA for $60 \mathrm{~min}$ at $37^{\circ} \mathrm{C}$ using a Promega M-MLV kit (Promega Corporation, Madison, WI, USA). For quantitative analysis of gene expression, RT-qPCR was conducted using a SYBR premix Ex Taq II kit (Takara Biotechnology Co., Ltd., Dalian, China) according to the manufacturer's protocols. The reaction mixture was subjected to RT-qPCR to detect levels of CCR7, ANO6, ERK1/2 and GAPDH. GAPDH was used as an endogenous reference. All primer sequences used for the amplification are listed in Table I. The PCR thermocycling conditions were as follows: Initial denaturation for $30 \mathrm{sec}$ at $95^{\circ} \mathrm{C}$, followed by 45 cycles of denaturation for $5 \mathrm{sec}$ at $95^{\circ} \mathrm{C}$ and annealing for $30 \mathrm{sec}$ at $60^{\circ} \mathrm{C}$. Following the last cycle, a final extension of $10 \mathrm{sec}$ at $60^{\circ} \mathrm{C}$ was completed and thereafter the samples were maintained at $4^{\circ} \mathrm{C}$. The relative expression level of the genes was calculated using the $2^{-\Delta \Delta \mathrm{Cq}}$ method (19).

Western blot analysis. BxPC-3-CCR7-GFP and BxPC-3-GFP cells were stimulated or not with CCL21 for $16 \mathrm{~h}$ at $37^{\circ} \mathrm{C}$ and the BxPC-3-CCR7-GFP cells in the absence of CCL21 were pretreated with $10 \mu \mathrm{mol} / 1 \mathrm{U} 0126$ for $2 \mathrm{~h}$ at $37^{\circ} \mathrm{C}$. Then the whole-cell protein and nuclear protein extracts from cells were prepared in an ice-cold lysis buffer (50 mM Tris, $\mathrm{pH} 6.8$, $0.2 \%$ SDS, $10 \%$ glycerol and $1 \%$ mercaptoethanol) for western blot analysis according to the manufacturers' protocols. Total protein was determined using BCA assay (Beyotime Institute of Biotechnology, Haimen, China). A total of $15 \mu \mathrm{g}$ protein were subjected to $10 \%$ SDS-PAGE and transferred onto polyvinylidene fluoride membranes. Following blocking for 
Table I. Primers for reverse transcription-quantitative polymerase chain reaction.

\begin{tabular}{lllc}
\hline Gene & \multicolumn{1}{c}{ Forward primer, 5'-3' } & \multicolumn{1}{c}{ Reverse primer, 5'-3' } & Product size, bp \\
\hline CCR7 & TGGTGGTGGCTCTCCTTG & CTGTGGTGTTGTCTCCGATG & 84 \\
ANO6 & GAACAAGCCCGACCAGAATAC & CCCAATAACTGAAGCGATGATC & 171 \\
ERK1 & ATGTCATCGGCATCCGAGAC & GGATCTGGTAGAGGAAGTAGCA & 156 \\
ERK2 & TTACGACCCGAGTGACGA & CTGTATCCTGGCTGGAATCT & 129 \\
GAPDH & TGACTTCAACAGCGACACCCA & CACCCTGTTGCTGTAGCCAAA & 121 \\
\hline
\end{tabular}

CCR7, C-C motif chemokine receptor 7; ANO6, anoctamin 6; ERK, extracellular signal-regulated kinase.

$1 \mathrm{~h}$ at room temperature with $5 \%$ skimmed milk in TBST, filters were incubated with the following primary antibodies for $2 \mathrm{~h}$ at room temperature: CCR7 (dilution, 1:5,000), ANO6 (dilution, 1:500), pERK antibody (dilution, 1:500) and GAPDH (dilution, 1:500), and were visualized with a horseradish peroxidase-conjugated secondary antibody rabbit $\mathrm{IgG}$ for $1.5 \mathrm{~h}$ at room temperature (cat no. sc-2004; dilution, 1:2,000; Santa Cruz Biotechnology, Inc., Dallas, TX, USA) and an ECL substrate detection ECL-PLUS/kit (Thermo Fisher Scientific, Inc.). Protein bands were quantified using ImageJ software version 1.49 (National Institutes of Health, Bethesda, MD, USA).

Statistical analysis. Each experiment was performed in triplicate and repeated as three independent experiments. Error bars represent the standard deviation. Statistical significance was determined using Student's t-test or one-way analysis of variance followed by Turkey's post-hoc test, $\mathrm{P}<0.05$ was considered to indicate a statistically significant difference.

\section{Results}

CCR7 overexpression promotes BxPC-3 cell migration. The present study evaluated the mRNA expression levels of CCR7 and the migratory capacities of three PDAC cell lines, BxPC-3, AsPC-1 and Panc-1. The results revealed that CCR7 was expressed in all the analyzed PDAC cell lines at the mRNA level and a positive association was observed between CCR7 expression and migratory capacities $(\mathrm{P}<0.01$; Fig. 1$)$.

To investigate the involvement of CCR7 in regulating the migration phenotype of PDAC cells in vitro, exogenous CCR7 was expressed in BxPC-3 cells via lentivirus transfection, as these cells exhibit relatively low levels of CCR7 expression and relatively poor migration ability. The lentivirus plasmid LV-CCR7-GFP contained a GFP reporter gene. The high mRNA and protein expression of CCR7 was detectable in BxPC-3-CCR7-GFP cells, compared with BxPC-3-GFP cells and BxPC-3 cells ( $\mathrm{P}<0.001$; Fig. 2). BxPC-3-CCR7-GFP cells exhibited a markedly increased migration ability compared with the BxPC-3-GFP cells $(\mathrm{P}<0.001$; Fig. 3$)$. These data indicated that $\mathrm{CCR} 7$ overexpression may promote migration in BxPC-3 cells.

CCR7 ligand-binding function affects BXPC-3 cell migration. To determine whether the ligand-binding ability of CCR7 affected BxPC-3 cell migration, BxPC-3-CCR7-GFP and
$\mathrm{BxPC}-3$

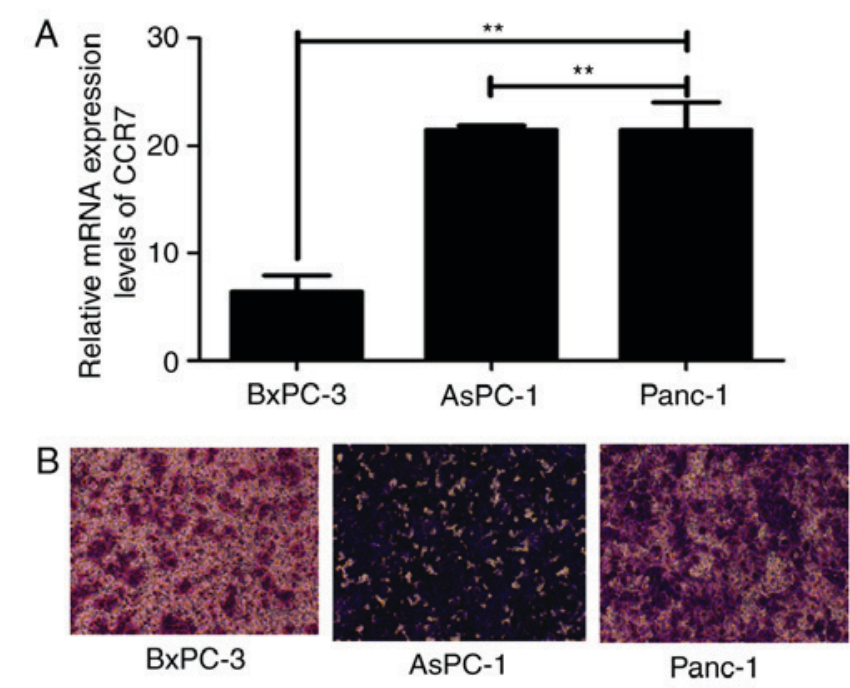

Figure 1. The association between CCR7 expression and metastatic potential in different PDAC cell lines. (A) Relative mRNA expression levels of CCR7 in three pancreatic cancer cell lines were detected by reverse transcription-quantitative polymerase chain reaction. (B) A transwell migration assay was used to evaluate the migration capacity of three PDAC cell lines (magnification, $\mathrm{x} 100){ }^{* *} \mathrm{P}<0.01$. CCR7, C-C motif chemokine receptor 7; PDAC, pancreatic ductal adenocarcinoma.

BxPC-3-GFP cells were pretreated with $100 \mathrm{ng} / \mathrm{ml}$ CCL21 for $16 \mathrm{~h}$. The binding of ligand and receptor resulted in an increase in migration, indicating that ligand-binding to CCR7 was positively associated with the migration of BxPC-3 cells $(\mathrm{P}<0.01$; Fig. 3$)$.

In addition, the effect of the CCL21/CCR7 axis on the proliferation of $\mathrm{BxPC}-3$ cells was also examined. The proliferation change of different pretreated BxPC-3 cells was determined using an MTT cell proliferation assay. The results demonstrated that the rate of proliferation of BxPC-3-CCR7-GFP cells may increase from the third day of the experiment compared with that of the BxPC-3-GFP cells $(\mathrm{P}<0.001$; Fig. 3). However, exogenous CCL21 did not significantly alter the cellular proliferation of either cell type. These results indicated that ligand binding to $\mathrm{CCR} 7$ is not associated with BxPC-3 cell proliferation.

CCR7 overexpression promotes $B x P C-3$ cell migration by inducing ERK pathway activity. A previous study reported that CCR7 pathway upregulates Twist expression via ERK signaling to manage the epithelial-mesencymal transition of 
A
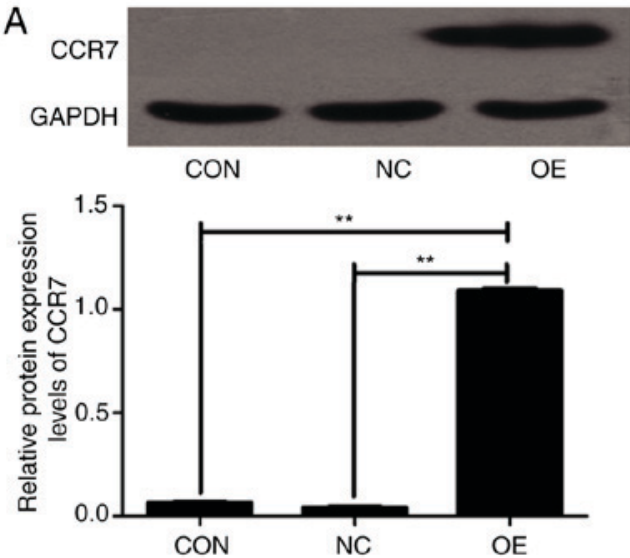

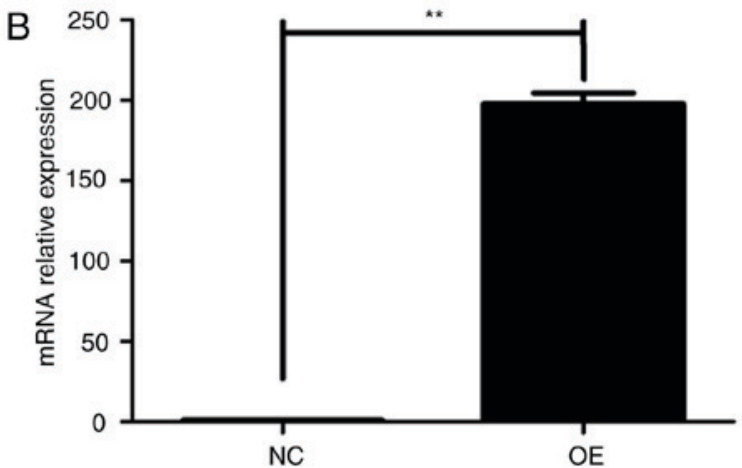

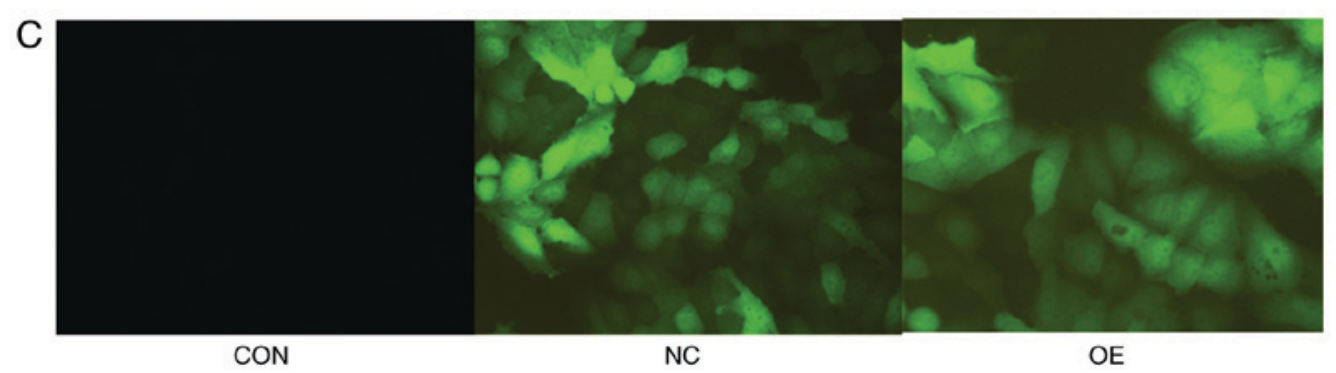

Figure 2. CCR7 overexpression in BxPC-3 cells. BxPC-3 cells were transfected with a lentivirus containing LV-CCR7-GFP or LV-GFP. (A) CCR7 protein expression in the whole cell lysates of CON, NC or OE cells was determined by western blot analysis. GAPDH was used as a loading control. (B) CCR7 mRNA expression in the whole cell lysates of $\mathrm{NC}$ and $\mathrm{OE}$ cells was determined using reverse transcription-quantitative polymerase chain reaction analysis. (C) CON, $\mathrm{NC}$ and OE cells under a fluorescent microscope, with $\sim 90 \%$ transfection efficiency (magnification, $\mathrm{x} 200$ ). ${ }^{* *} \mathrm{P}<0.01$. CCR7, C-C motif chemokine receptor 7 ; GFP, green fluorescent protein; CON, untransfected control; NC, blank-vector-transfected negative control; OE, CCR7-overexpressing cells.
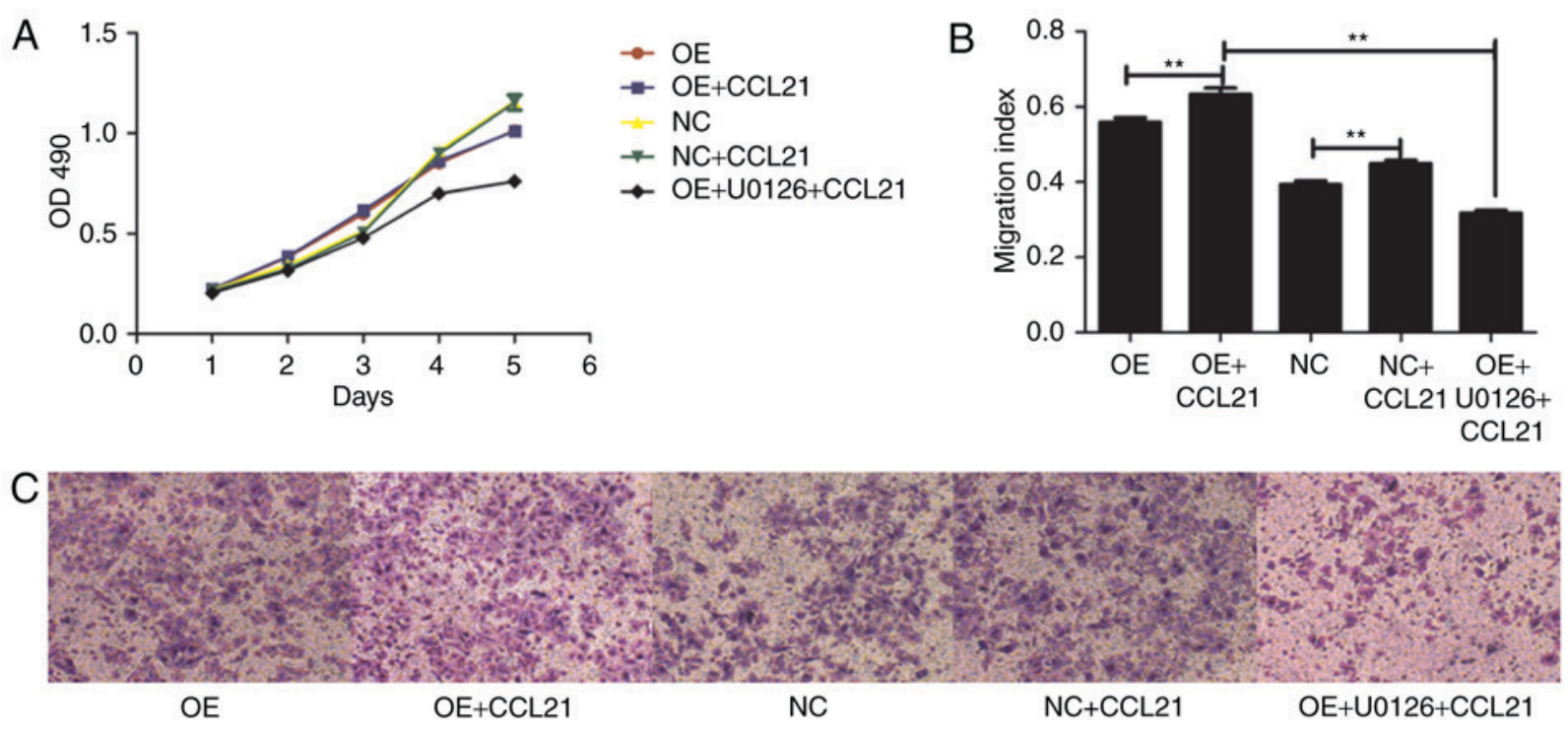

Figure 3. Effects of CCL21/CCR7 axis and the extracellular signal-regulated kinase inhibitor, U0126, on proliferation and migration. OE cells were pretreated with or without U0126 for $2 \mathrm{~h}$, followed by stimulation with CCL21 for $16 \mathrm{~h}$. (A) Proliferative ability of OE cells and NC cells pretreated with or without CCL21 and U0126. (B) Migratory ability of OE and NC cells pretreated with or without CCL21 and U0126. (C) Transwell migration assay was used to evaluate the migration capacity of different cells (magnification, $\mathrm{x} 100$ ). ${ }^{* *} \mathrm{P}<0.01$. CCL21, C-C motif chemokine ligand 21; CCR7, C-C motif chemokine receptor 7; OD, optical density; CON, untransfected control; NC, blank-vector-transfected negative control; OE, CCR7-overexpressing cells.

PDAC (20). To investigate whether the ERK1/2 pathway is downstream activated by CCL21-CCR7 binding, the phosphorylation of ERK1/2 was observed for the entire duration of stimulation with CCL21. To assess whether the phosphorylation of ERK1/2 was dependent on the activation of CCR7, the cells were pre-incubated with the ERK1/2 specific inhibitor
U0126. Furthermore, BxPC-3-CCR7-GFP cells underwent migration assays adding CCL21 as a chemoattractant into the lower chamber of the transwell chamber in the presence or absence of U0126. As demonstrated in Fig. 3, according to the transwell assay, the number of migrated cells was significantly decreased following pre-incubation with U0126 ( $<<0.001)$. 
A

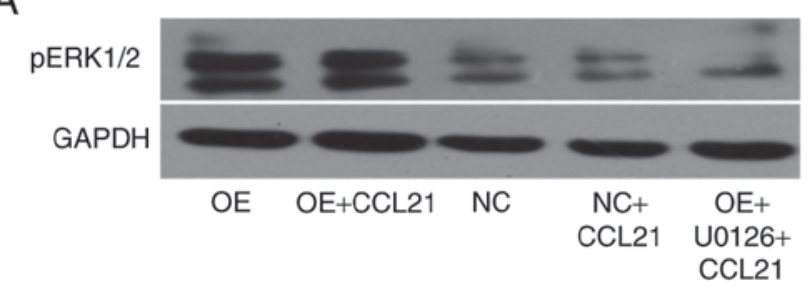

B
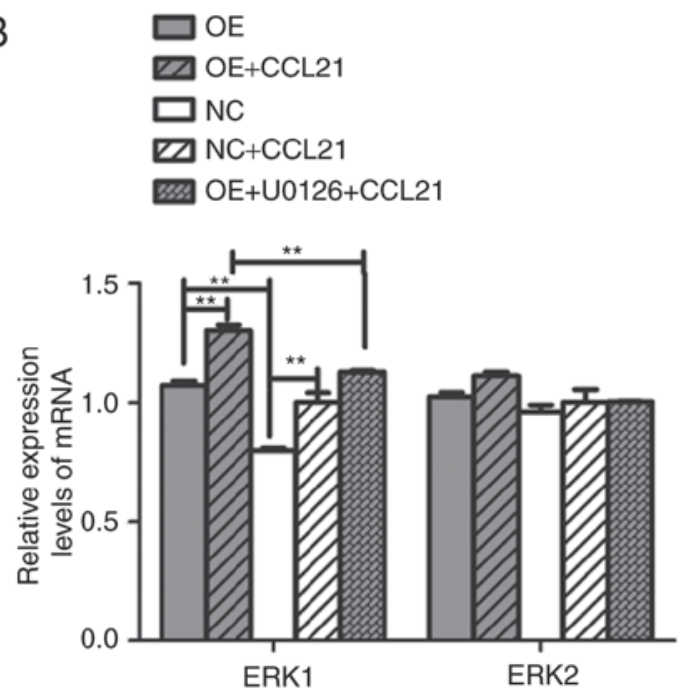

Figure 4. CCR7 regulates ERK expression in different cells. (A) Western blot analysis of pERK in OE and NC cells pretreated with or without CCL21 and U0126. GAPDH was used as a loading control. (B) Transcription levels of ERK1/2 in OE and NC cells with or without pretreatment with CCL21 and U0126. ${ }^{* *} \mathrm{P}<0.01$. CCR7, C-C motif chemokine receptor 7; ERK, extracellular signal-regulated kinase; pERK, phosphorylated ERK; CCL21, C-C motif chemokine ligand 21; NC, blank-vector-transfected negative control; OE, CCR7-overexpressing cells.

A
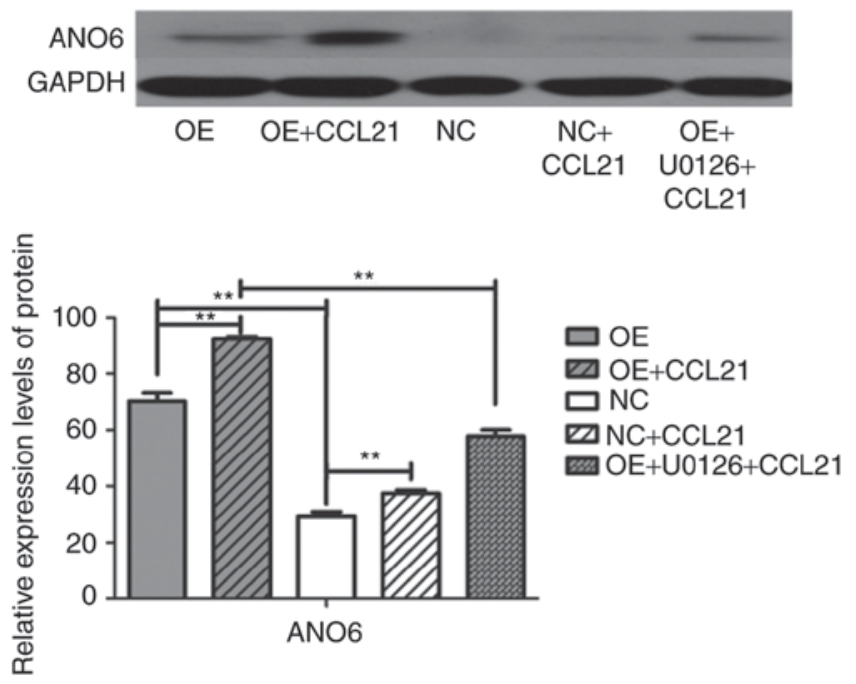

B

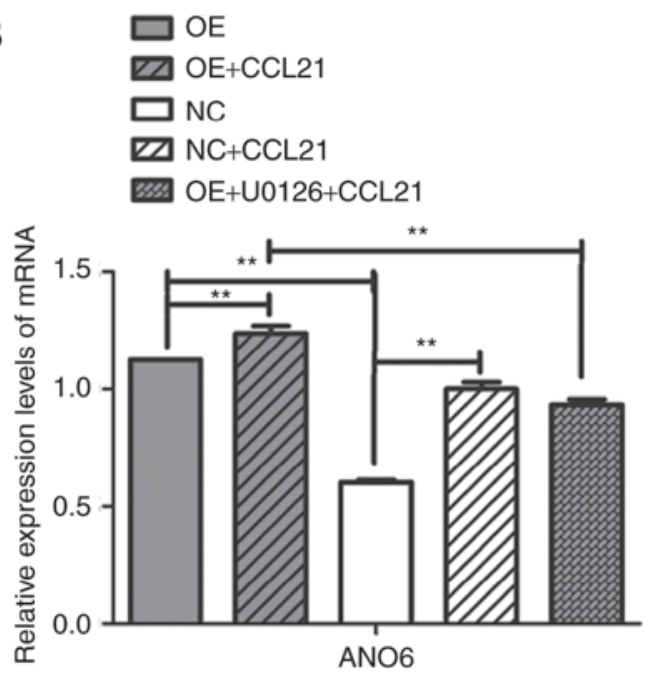

Figure 5. CCR7 ligand-binding function modulates BxPC-3 cell migration by regulating ANO6 expression. (A) Western blot analysis of ANO6 in OE and NC cells pretreated with or without CCL21 and U0126. GAPDH was used as a loading control. (B) Reverse transcription-quantitative polymerase chain reaction analysis of ANO6 in OE and NC cells with or without pretreatment of CCL21 and U0126. ${ }^{* *} \mathrm{P}<0.01$. CCR7, C-C motif chemokine receptor 7; CCL21, C-C motif chemokine ligand 21; ANO6, anoctamin 6; NC, blank-vector-transfected negative control; OE, CCR7-overexpressing cells.

The level of cellular proliferation was also inhibited by U0126 significantly $(\mathrm{P}<0.001$; Fig. 3). These results indicate that ERK serves a notable role in the response of BxPC-3 cells to CCR7. To confirm further whether CCR7 regulates the migration of BxPC-3 cells by modulating ERK expression, the mRNA and protein levels of ERK in different cell lines were analyzed. It was revealed that overexpression of CCR7 and the binding of CCL21 to CCR7 upregulated the gene expression level of ERK1 and the level of pERK protein in BxPC-3-CCR7-GFP cells compared with BxPC-3-GFP cells ( $<<0.001$; Fig. 4), but this had no influence on the gene expression level of ERK2 ( $\mathrm{P}>0.05)$. Following the pre-incubation of BxPC-3-CCR7-GFP cells with U0126 for $2 \mathrm{~h}$, the ERK1 gene and levels of pERK protein decreased, as determined by RT-qPCR and western blot analysis $(\mathrm{P}<0.001$; Fig. 4). Pre-incubation with U0126 inhibited the influence of CCL21/CCR7 on the expression of ERK. These findings indicated that CCL21 drives increases in BxPC-3 cell migration through the ERK pathway.

Involvement of the ERK signaling pathway in CCR7 ligand-mediated upregulation of ANO6. To investigate the role of CCL21/CCR7 binding in ANO6 production, the constitutive expression of CCR7 was screened in different PDAC cell lines and a gain-of-function model was developed. A high level of CCR7 expression was associated with the significant upregulation of ANO6 mRNA and protein expression (Fig. 5; $\mathrm{P}<0.001$ ). To determine whether CCL21/CCR7 interaction regulated the expression of ANO6, 
BxPC-3 cells were incubated with or without human CCL21 for $16 \mathrm{~h}$. These results demonstrated that ANO6 expression mRNA and protein levels were significantly higher in the CCL21-treated cells than in the untreated cells. Overall, these findings indicated that CCL21/CCR7 binding has the potential to regulate ANO6 expression.

Finally, to assess the functional roles of the ERK1/2 pathway in CCL21-induced ANO6 secretion, BxPC-3 cells were pre-treated with the ERK1/2 inhibitor U0126 prior to treatment with CCL21 for $16 \mathrm{~h}$. Pre-treatment with the ERK1/2 inhibitor yielded a significantly reduced ANO6 expression when compared with CCL21 treatment alone (Fig. 5; P <0.001). Therefore, it can be concluded that activation of the ERK1/2 signaling pathway downstream of CCR7 is involved in the regulation of ANO6 expression.

\section{Discussion}

Metastasis is the leading cause of cancer-associated mortality; this is particularly true for PDAC, owing to its highly invasive and metastatic behavior (21). Further understanding of the mechanisms underlying aggressive cancer cell phenotypes, including knowledge of this invasive, metastatic behavior, is required if patient outcomes are to be improved (22).

The metastasis of tumor cells to the lymph nodes is a complex process. Previous studies have indicated that the CCL21/CCR7 axis serves a pivotal role in triggering lymphatic metastasis of PDAC $(1,10,23)$. The aim of the present study was to determine whether the CCL21/CCR7 axis is associated with BxPC-3 cell proliferation and migration by upregulating the expression of ANO6 via the ERK signaling pathway.

To investigate the mechanism of CCR7 promotion on PDAC cell lines, the expression of CCR7 in different PDAC cell types was examined and a gain-of-function model comprising BxPC-3-CCR7-GFP cells was generated. Stable CCR7 overexpression led to an enhancement of the migratory ability of BxPC-3 cells, but elicited no effect on BxPC-3 cell proliferation. These results further supported the hypothesis that CCR7 may serve a role in pancreatic cancer metastasis. However, the molecular mechanisms by which CCR7 regulates cell migration remain unclear. To determine how CCR7 promotes BxPC-3 cell migration, the present study focused on delineating the association between CCR7 and ANO6.

Autocrine CCR7 stimulation by endogenous CCL21 may present a possible mechanism to enhance migration in PDAC cells, a finding that was consistent with the findings of Sperveslage et al (24). Additionally, following blocking with a CCR7 antibody, a significant decrease in bladder cancer cells was observed (25). However, the interactions between chemokines and their receptors are fundamental to tumor cell motility and migration. Therefore, CCL21 may activate CCR7 to enhance BxPC-3 cell migration.

The ANO family members are accessible cell surface proteins that are upregulated in tumors and are therefore potential targets for therapeutic antibodies and as biomarkers for tumors (26). ANO6 was activated upon the interaction of CCL21 with CCR7 and serves an important role in chemokine-induced dendritic cell migration. In a transwell migration assay, the chemokine-induced migration of immature and mature dendritic cells was markedly impaired by treatment with a small interfering RNA against ANO6 (27). However, the effect of ANO6 on PDAC cells remains unknown. Whether ANO6 mediates the migration of human PDAC cells and whether the transcription expression levels are promoted by CCR7 requires further investigation. RT-qPCR and western blot analysis confirmed that the ANO6 mRNA and protein expression levels were upregulated, which was coupled with increased ANO6 activity in BxPC-3-CCR7-GFP cells, compared with that in the $\mathrm{NC}$ cells. Additionally, it was revealed that CCR7 promoted the expression of ANO6 mRNA and protein when BxPC-3 cells were incubated with CCL21 for $16 \mathrm{~h}$.

It is known that ERK, as a mitogen-activated protein kinase signal transduction pathway, is modulated by the activity of CCR7 (28-32). The present study demonstrated that overexpression of CCR7 significantly increased the gene and protein expression levels of ERK. When BxPC-3 cells were pretreated with the ERK inhibitor U0126, the migration of BxPC-3 cells was reduced and the mRNA transcription levels and protein expression levels of ANO6 and pERK in BxPC-3 cells were decreased. Therefore, it is likely that the ERK signaling pathway is associated with CCR7-dependent cell motility, including regulation of ANO6 transcription.

In conclusion, to the best of our knowledge, the present study is the first to indicate that CCL21/CCR7 upregulates ANO6 via ERK to promote BxPC-3 cell migration, which indicates that CCL21/CCR7 and ANO6 may serve crucial roles in the lymphatic metastasis of PDAC. Further animal studies are required to elucidate the sequence of events leading to the CCR7-mediated metastatic phenotype, and will enable the development of therapeutic strategies aimed at blocking these carcinogenic and metastatic effects.

\section{Acknowledgements}

Not applicable.

\section{Funding}

The present study was supported by the Shanghai Municipal Commission of Health and Family Planning Grant of China (grant no. 955), the Shanghai Jiao Tong University Affiliated Shanghai Sixth People's Hospital Grant of China (grant no. 1581) and the Science and Technology Innovation Special Fund of Shanghai Jiao Tong University Shanghai Jiao Tong University (grant no. YG2016QN18).

\section{Availability of data and materials}

All data generated or analyzed during this study are included in this published article.

\section{Authors' contributions}

LW performed the cellular functional experiments and was a major contributor in writing the manuscript. XYZ performed the cell culture. JSZ and NWC provided advice about the conception and design, directed the experiment and revised the manuscript critically for important intellectual content. HNF performed the reverse transcription quantitative polymerase chain reaction. WY performed the western blotting. 
JHG performed transfection of the cells, data analysis, writing the manuscript and was committed to funding the experiment. All authors have read and approved the final version of the manuscript.

\section{Ethics approval and consent to participate}

Not applicable.

\section{Patient consent for publication}

Not applicable.

\section{Competing interests}

All authors declare that they have no competing interests.

\section{References}

1. Neoptolemos JP, Stocken DD, Friess H, Bassi C, Dunn JA, Hickey H, Beger H, Fernandez-Cruz L, Dervenis C, Lacaine F, et al: A randomized trial of chemoradiotherapy and chemotherapy after resection of pancreatic cancer. N Eng1 J Med 350: 1200-1210, 2004.

2. Zlotnik A and Yoshie O: Chemokines: A new classification system and their role in immunity. Immunity 12: 121-127, 2000.

3. Ben-Baruch A: The multifaceted roles of chemokines in malignancy. Cancer Metastasis Rev 25: 357-371, 2006.

4. Zlotnik A: Chemokines and cancer. Int J Cancer 119: 2026-2029, 2006.

5. Ding Y, Shimada Y, Maeda M, Kawabe A, Kaganoi J, Komoto I, Hashimoto $\mathrm{Y}$, Miyake $\mathrm{M}$, Hashida $\mathrm{H}$ and Imamura $\mathrm{M}$ Association of CC chemokine receptor 7 with lymph node metastasis of esophageal squamous cell carcinoma. Clin Cancer Res 9: 3406-2412, 2003.

6. Tutunea-Fatan E, Majumder M, Xin X and Lala PK: The role of CCL21/CCR7 chemokine axis in breast cancer-induced lymphangiogenesis. Mol Cancer 14: 35, 2015.

7. Irino $T$, Takeuchi $H$, Matsuda $S$, Saikawa $Y$, Kawakubo $H$, Wada N, Takahashi T, Nakamura R, Fukuda K, Omori T and Kitagawa Y: CC-Chemokine receptor CCR7: A key molecule for lymph node metastasis in esophageal squamous cell carcinoma. BMC Cancer 14: 291, 2014.

8. Oliveira-Neto HH, de Souza PP, da Silva MR, Mendonça EF, Silva TA and Batista AC: The expression of chemokines CCL19, CCL21 and their receptor CCR7 in oral squamous cell carcinoma and its relevance to cervical lymph node metastasis. Tumor Biol 34: 65-70, 2013.

9. Al-Shareef H, Hiraoka SI, Tanaka N, Shogen Y, Lee AD, Bakhshishayan S and Kogo M: Use of NRP1, a novel biomarker, along with VEGF-C, VEGFR-3, CCR7 and SEMA3E, to predict lymph node metastasis in squamous cell carcinoma of the tongue. Oncol Rep 36: 2444-2454, 2016.

10. Guo J, Lou W, Ji Y and Zhang S: Effect of CCR7, CXCR4 and VEGF-C on the lymph node metastasis of human pancreatic ductal adenocarcinoma. Oncol Lett 5: 1572-1578, 2013.

11. Li J, Sun R, Tao K and Wang G: The CCL21/CCR7 pathway plays a key role in human colon cancer metastasis through regulation of matrix metalloproteinase-9. Dig Liver Dis 43: 40-47, 2011

12. Ma H, Gao L, Li S, Qin J, Chen L, Liu X, Xu P, Wang F, Xiao H, Zhou S, et al: CCR7 enhances TGF- $\beta 1$-induced epithelial-mesenchymal transition and is associated with lymph node metastasis and poor overall survival in gastric cancer. Oncotarget 6: 24348-24360, 2015.

13. Liu FY, Safdar J, Li ZN, Fang QG, Zhang X, Xu ZF and Sun CF: CCR7 regulates cell migration and invasion through JAK2/STAT3 in metastatic squamous cell carcinoma of the head and neck. Biomed Res Int 2014: 415375, 2014.
14. Liu FY, Safdar J, Li ZN, Fang QG, Zhang X, Xu ZF and Sun CF: CCR7 regulates cell migration and invasion through MAPKs in metastatic squamous cell carcinoma of head and neck. Int J Oncol 45: 2502-2510, 2014.

15. Kunzelmann K,Nilius B, Owsianik G, Schreiber R, Ousingsawat J, Sirianant L, Wanitchakool P, Bevers EM and Heemskerk JW: Molecular functions of anoctamin 6 (TMEM16F): A chloride channel, cation channel, or phospholipid scramblase? Pflugers Arch 466: 407-414, 2014.

16. Miettinen $M$ : Immunohistochemistry of soft tissue tumours-review with emphasis on 10 markers. Histopathology 64: 101-118, 2014.

17. Jacobsen KS, Zeeberg K, Sauter DR, Poulsen KA, Hoffmann EK and Schwab A: The role of TMEM16A (ANO1) and TMEM16F (ANO6) in cell migration. Pflugers Arch 465: 1753-1762, 2013.

18. Wu S, Xing W, Peng J, Yuan X, Zhao X, Lei P, Li W, Wang M, Zhu H, Huang B, et al: Tumor transfected with CCL21 enhanced reactivity and apoptosis resistance of human monocyte-derived dendritic cells. Immunobiology 213: 417-426, 2008.

19. Livak KJ and Schmittgen TD: Analysis of relative gene expression data using real-time quantitative PCR and the 2(-Delta Delta C(T)) method. Methods 25: 402-408, 2001.

20. Li K, Xu B, Xu G and Liu R: CCR7 regulates Twist to induce the epithelial-mesenchymal transition in pancreatic ductal adenocarcinoma. Tumour Biol 37: 419-424, 2016.

21. Vincent A, Herman J, Schulick R, Hruban RH and Goggins M: Pancreatic cancer. Lancet 378: 607-620, 2011.

22. Giovannetti E, van der Borden CL, Frampton AE, Ali A, Firuzi $\mathrm{O}$ and Peters GJ: Never let it go: Stopping key mechanisms underlying metastasis to fight pancreatic cancer. Semin Cancer Biol 44: 43-59, 2017.

23. Nakata B, Fukunaga S, Noda E, Amano R, Yamada $\mathrm{N}$ and Hirakawa K: Chemokine receptor CCR7 expression correlates with lymph node metastasis in pancreatic cancer. Oncology 74 : 69-75, 2008.

24. Sperveslage J, Frank S, Heneweer C, Egberts J, Schniewind B Buchholz M, Bergmann F, Giese N, Munding J, Hahn SA, et al: Lack of CCR7 expression is rate limiting for lymphatic spread of pancreatic ductal adenocarcinoma. Int J Cancer 131: E371-E381, 2012.

25. Mo M, Zhou M, Wang L, Qi L, Zhou K, Liu LF, Chen Z and $\mathrm{Zu}$ XB: CCL21/CCR7 enhances the proliferation, migration, and invasion of human bladder cancer T24 cells. PLoS One 10: e0119506, 2015.

26. Shang L, Hao JJ, Zhao XK, He JZ, Shi ZZ, Liu HJ, Wu LF, Jiang YY, Shi F, Yang H, et al: ANO1 protein as a potential biomarker for esophageal cancer prognosis and precancerous lesion development prediction. Oncotarget 7: 24374-24382, 2016.

27. Szteyn K, Schmid E, Nurbaeva MK, Yang W, Münzer P, Kunzelmann K, Lang F and Shumilina E: Expression and functional significance of the $\mathrm{Ca}(2+)$-activated $\mathrm{Cl}(-)$ channel ANO6 in dendritic cells. Cell Physiol Biochem 30: 1319-1332, 2012.

28. Cheng S, Guo J, Yang Q and Yang X: Crk-like adapter protein regulates CCL19/CCR7-mediated epithelial-to-mesenchymal transition via ERK signaling pathway in epithelial ovarian carcinomas. Med Oncol 32: 47, 2015.

29. Zhang J, Zhou Y and Yang Y: CCR7 pathway induces epithelial-mesenchymal transition through up-regulation of Snail signaling in gastric cancer. Med Oncol 32: 467, 2015.

30. Li G, Yang Y, Xu S, Ma L, He M and Zhang Z: Slug signaling is up-regulated by CCL21/CCR7 [corrected] to induce EMT in human chondrosarcoma. Med Oncol 32: 478, 2015.

31. Su ML, Chang TM, Chiang CH, Chang HC, Hou MF, Li WS and Hung WC: Inhibition of chemokine (C-C motif) receptor 7 sialylation suppresses CCL19-stimulated proliferation, invasion and anti-anoikis. PLoS One 9: e98823, 2014.

32. Zhang L, Wang D, Li Y, Liu Y, Xie X, Wu Y, Zhou Y, Ren J, Zhang J, Zhu H and Su Z: CCL21/CCR7 axis contributed to CD133+ pancreatic cancer stem-like cell metastasis via EMT and Erk/NF-kB pathway. PLoS One 11: e0158529, 2016. 